\title{
Sex-specific prediction value of common carotid artery diameter for stroke risk in a hypertensive population: a cross-sectional study
}

\author{
Weishuang Xue ${ }^{1 \#}$, Yuanmeng Tian ${ }^{2,3 \#}$, Li Jing ${ }^{2,3}$, Guangxiao Li ${ }^{4}$, Han Yan ${ }^{2,3}$, Boqiang Zhang ${ }^{2,3}$, Liying Xing ${ }^{2,3}$, \\ Shuang Liu,
}

${ }^{1}$ Department of Neurology, The First Hospital of China Medical University, Shenyang, China; ${ }^{2}$ Institute of Preventive Medicine, China Medical University, Shenyang, China; ${ }^{3}$ Department of Chronic Disease Preventive and Control, Liaoning Provincial Center for Disease Control and Prevention, Shenyang, China; ${ }^{4}$ Department of Medical Record Management Center, The First Hospital of China Medical University, Shenyang, China; ${ }^{5}$ Department of Cardiovascular Ultrasound, The First Hospital of China Medical University, Shenyang, China; ${ }^{6}$ Department of Ultrasound, The Fourth Affiliated Hospital of China Medical University, Shenyang, China

Contributions: (I) Conception and design: S Liu, L Xing; (II) Administrative support: S Liu, L Xing; (III) Provision of study materials or patients: L Jing, G Li, H Yan, B Zhang; (IV) Collection and assembly of data: Y Tian, W Xue, L Jing, G Li, H Yan, B Zhang; (V) Data analysis and interpretation: Y Tian, W Xue, L Jing, G Li, H Yan, B Zhang; (VI) Manuscript writing: All authors; (VII) Final approval of manuscript: All authors.

\#These authors contributed equally to this work.

Correspondence to: Shuang Liu, MD, PhD. Department of Cardiovascular Ultrasound, The First Hospital of China Medical University, Shenyang, China; Department of Ultrasound, The Fourth Affiliated Hospital of China Medical University, Shenyang, China. Email: liushuang_cmu1h@163.com.

Background: The importance of sex as a risk factor for stroke has been established. This study aimed to assess sex-related disparities in carotid artery diameter and stroke in a hypertensive population.

Methods: The cross-sectional survey was conducted in rural areas of northeast China. A multistage cluster sampling method was employed to select a representative population. The study comprised 3,245 individuals with hypertension. The common carotid artery (CCA) interadventitial diameter was measured by ultrasound. A linear model of restricted cubic spline function was used to characterize the concentration-response (C-R) relationship between CCA diameter and stroke.

Results: The overall prevalence of stroke was $8.9 \%$ among hypertensive individuals, with a higher rate in men than in women $(10.8 \%$ vs. $7.6 \%)$. When the women's CCA diameters were divided into quartiles, the top quartile $(>8.10 \mathrm{~mm}$ ) had a 2.49 (95\% CI: 1.36-4.56) times greater risk of stroke compared to the bottom quartile $(\leq 6.80 \mathrm{~mm})$ after adjustment was made for other variables. The C-R relationship further confirmed a positive association between CCA diameter and stroke prevalence in women. Moreover, a category-free net reclassification index $(0.325 ; 95 \% \mathrm{CI}: 0.173-0.476 ; \mathrm{P}<0.001)$ and an integrated discrimination index $(0.008$; 95\% CI: $0.004-0.012, \mathrm{P}<0.001)$ showed improvement in predicting the probability of stroke from CCA diameter. However, no significant relationship between CCA diameter and prevalence of stroke was found in men.

Conclusions: The risk of stroke increased proportionally with the enlargement of the CCA diameter in women, supporting the sex-specific value of CCA diameter in optimizing the risk stratification of stroke.

Keywords: Carotid arteries; stroke; risk factors; hypertension

Submitted Jun 09, 2021. Accepted for publication Oct 09, 2021.

doi: 10.21037/qims-21-598

View this article at: https://dx.doi.org/10.21037/qims-21-598 


\section{Introduction}

Stroke remains the second leading cause of death and the major cause of disability globally, incurring a considerable societal burden in terms of productivity losses and health care costs (1). As a highly preventable disorder, the incidence of stroke could be reduced by approximately $75 \%$ by controlling risk factors (2). Hypertension is the most important modifiable risk factor for stroke, and the risk of stroke increases steeply with increases in diastolic and systolic blood pressure (3). Hypertension often coexists and interacts with other risk factors such as atherosclerosis, which further increase stroke risk and worsen stroke outcomes. Therefore, early screening and prevention of stroke in hypertensive populations could yield considerable benefits and should be emphasized.

Hypertension is also a known risk factor of atherosclerosis and plays an important role in atherogenesis and endothelial dysfunction (4). Vascular remodeling is an important stage in the process of atherosclerosis (5). It usually manifests as carotid artery diameter enlargement, reflecting arterial structural changes over time or in response to risk factors, including hypertension (6). Therefore, carotid diameter enlargement, measured at the common carotid artery (CCA), has been hypothesized to be a surrogate marker of atherosclerosis in predicting stroke in the general population (7).

Furthermore, sex-related disparities in stroke and related risk factors have been consistently confirmed (8). Women tend to have a higher lifetime risk of stroke than do men (9). In addition, previous studies have reported that an enlarged CCA diameter is related to the incidence of adverse cardiovascular outcomes, including stroke, cardiovascular diseases, and mortality; however, they failed to adjust for important potential confounders such as sex $(7,10)$. As differences in CCA diameter related to ethnicity and sex have previously been observed, reported associations may not be valid for the Chinese population (11).

Therefore, the exact association between carotid artery enlargement and stroke among a hypertensive population remains uncertain. To optimize the risk stratification of stroke in hypertensive individuals, the present study aimed to estimate the sex-specific effect of carotid arterial enlargement on the prevalence of stroke among a hypertensive population and to assess the incremental value of carotid artery enlargement. We present the following article in accordance with the STROBE reporting checklist (available at https://dx.doi.org/10.21037/qims-21-598).

\section{Methods}

\section{Data collection and definitions}

This study was a cross-sectional survey conducted in rural areas of northeast China between September 2017 and May 2018. A multistage, geographically stratified random cluster sampling method was employed to ensure the selected study population was representative (12). In brief, a total of 13 villages were randomly selected from 2 counties (Chaoyang and Lingyuan) of Liaoning Province, and all permanent residents aged over 40 years $(n=6,830)$ were recognized as eligible participants, excepting those with pregnancy, cancer, or mental health disorders (13). We enrolled 5,838 participants (response rate: $85.5 \%$ ) in the study. Following the 2010 Chinese guidelines for the management of hypertension, hypertension was diagnosed in individuals who met 1 of the following criteria: mean systolic blood pressure (SBP) $\geq 140 \mathrm{mmHg}$, mean diastolic blood pressure (DBP) $\geq 90 \mathrm{mmHg}$, or self-reported use of antihypertensive drugs in the past 2 weeks (14). All the participants enrolled in the study were measured 3 times at 2-minute intervals after more than 5 minutes of rest in a seated position. We finally included 3,245 hypertensive participants in the study. The study was conducted in accordance with the Declaration of Helsinki (as revised in 2013). The study protocol was approved by the central ethics committee of the China National Center for Cardiovascular Disease. Written consent was obtained from all participants. If the participants were unable to provided signed consent, their proxies provided written informed consent for them.

For data collection, the following related risk factors were defined: diabetes [fasting plasma glucose (FPG) $\geq 7.0 \mathrm{mmol} / \mathrm{L}$ or glycated hemoglobin (HbA1c) $\geq 6.5 \%$ and/or selfreported diagnosis as previously determined by a physician], dyslipidemia [serum total cholesterol level $\geq 6.22 \mathrm{mmol} / \mathrm{L}$, or serum low-density lipoprotein cholesterol (LDL-C) level $\geq 4.14 \mathrm{mmol} / \mathrm{L}$, or serum triglyceride level $\geq 2.27 \mathrm{mmol} / \mathrm{L}$, or serum high-density lipoprotein cholesterol (HDL-C) level $<1.04 \mathrm{mmol} / \mathrm{L}$, or self-reported use of lipidregulating medications], lack of exercise (no moderateintensity exercise or less than the equivalent to walking for $\geq 30$ minutes, $\geq 3$ times per week), current smoking habit ( $\geq 1$ cigarette/day for at least 6 months), and excess weight or obesity [body mass index (BMI) $\geq 24 \mathrm{~kg} / \mathrm{m}^{2}$ ], all of which have been consistently described in previous studies $(15,16)$.

Stroke was defined following the World Health Organization recommendation (17), and the pointprevalence date was set as August 31, 2017. New-onset 
strokes in the 8-month survey were not included in the stroke prevalence calculation. Participants with uncertain histories of stroke were further assessed by a welltrained neurologist (18). Clinical records and computed tomography (CT) scans and/or magnetic resonance imaging (MRI) scans obtained during each participant's hospital stay were retrieved and carefully reviewed (12).

\section{Carotid ultrasonography}

Carotid ultrasound was performed using high-resolution B-mode ultrasonography (Mindray M7, Shenzhen, China) with a broadband $7 \mathrm{~L} 4 \mathrm{~S}$ linear array transducer. The procedure was performed by certified sonographers with $>3$ years of experience in vascular ultrasound imaging. All participants were examined in the supine position, and both left and right carotids were scanned.

For each participant, images of the right and left CCAs were centered 10-15 mm below the CCA bulb. The smallest diameter of the artery (end-diastolic image) was captured. Measurements were made with an edge detector that identified the media-adventitia interfaces of the near and far walls. Once the CCA measurement was taken on each side, the average values of the left and right sides were calculated and used for analysis. Fifty participants were randomly selected for the intraobserver variability of the CCA diameter. Variability was determined by calculating the coefficients of variation, which were calculated as the standard deviation of differences. A paired $t$ test was employed to test the difference between the 2 measurements.

\section{Statistical analysis}

Descriptive statistics were calculated for all variables. Continuous variables with normal distribution are summarized as means and standard deviations and expressed as medians and interquartile ranges. Differences between groups were compared using a $\chi^{2}$ test for categorical variables. Univariate and stepwise multivariate logistic regression analyses were employed to assess the relationship between carotid parameters and the prevalence of stroke. A category-free net reclassification index (NRI) and an integrated discrimination index (IDI) were used to assess the potential value of the CCA diameter in refining the risk stratification of stroke. A restricted cubic spline function linear model was used to characterize the concentrationresponse (C-R) relationship between CCA diameter and stroke prevalence (with 95\% CI). In accordance with our previous study, we chose CCA diameter levels that were encoded using a restricted cubic spline function with 3 knots, located in the 5th, 50th, and 95th percentiles (19). Statistical analyses were conducted using SPSS v. 22.0 (IBM Corp., Armonk, NY, USA), the R statistical software package (http://www.R-project.org, The R Foundation, Vienna, Austra), and SAS 9.4 software (SAS Institute, Cary, NC, USA). P values $<0.05$ were considered statistically significant.

\section{Results}

\section{Characteristics of the hypertensive population}

A flowchart of the population selection process for the study is shown in Figure 1. The characteristics of the hypertensive population are shown in Table 1 . The study population comprised 3,245 hypertensive individuals, including 1,301 $(40.1 \%)$ men and 1,944 women $(59.9 \%)$. The overall prevalence of stroke was $8.9 \%$. Prevalence was higher in men than in women (10.8\% vs. $7.6 \%)$.

Stroke and nonstroke groups among the hypertensive population were compared. Among the women, individuals with stroke had a significantly larger CCA diameter, higher systolic blood pressure, and a higher HbAlc level. However, no significant differences were found in BMI or levels of LDL-C or HDL-C between the stroke and nonstroke groups. Among the men, there were no significant differences in CCA diameter measurements between the stroke and nonstroke groups. The CCA diameters measured in the male nonstroke group were higher than those in the female nonstroke group $(\mathrm{P}<0.001)$. Participant age in these 2 groups also presented significant differences $(\mathrm{P}<0.01)$. There were no significant differences in CCA diameter and age between the male and female stroke groups. Intraobserver variability analysis revealed that the correlation coefficient between the first and second CCA diameter measurements was $0.92(\mathrm{P}>0.05)$, with no significant difference. The mean difference was $0.62 \mathrm{~mm}$ (standard deviation $0.25 \mathrm{~mm}$ ).

\section{Relationship between CCA diameter and prevalence of stroke}

Multivariate logistic regression was employed to demonstrate the relationship between CCA diameter and prevalence of stroke in men and women (Table 2). We 


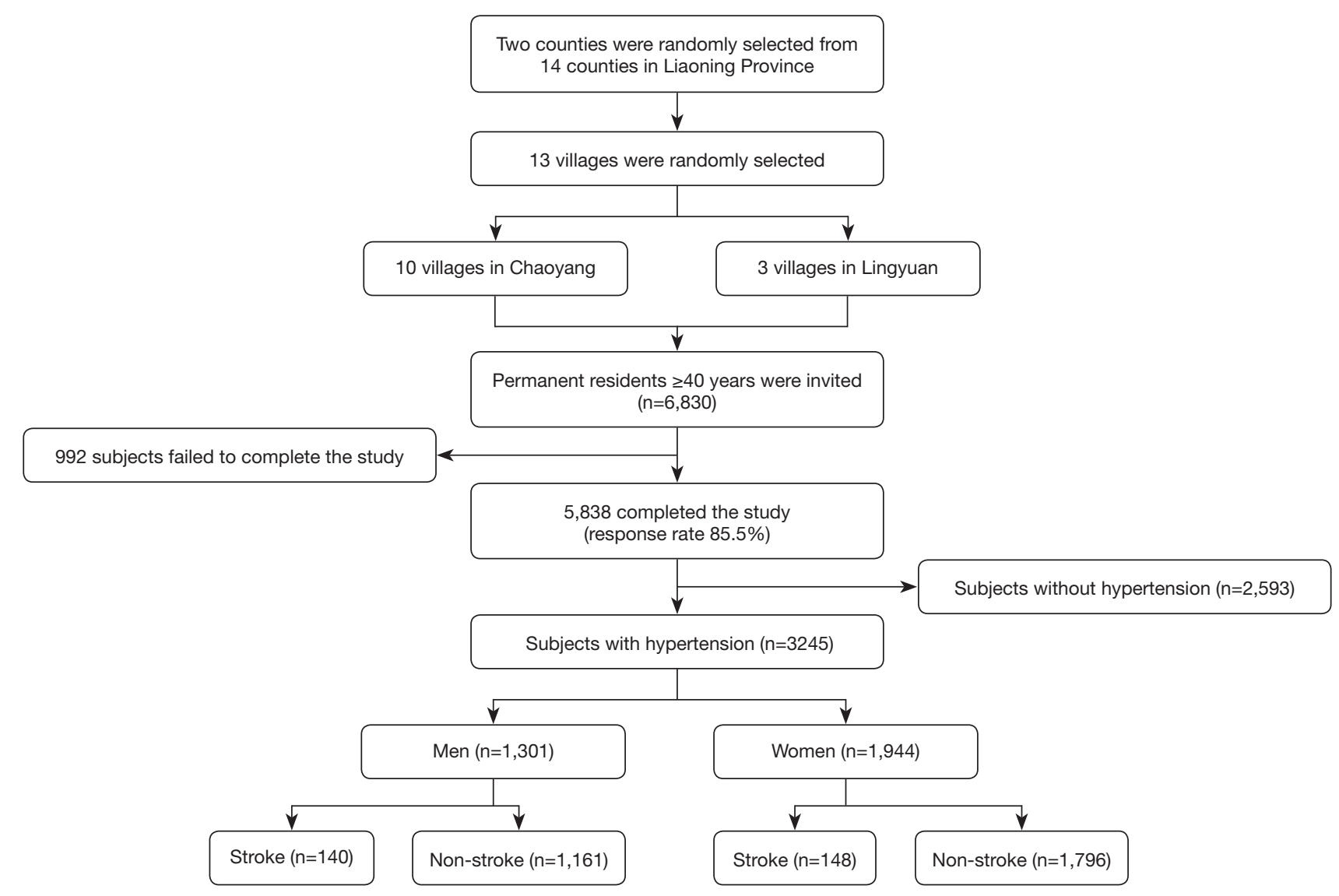

Figure 1 Flowchart of population selection.

divided the CCA diameters into quartiles. Among the women, the CCA diameters were $\leq 6.80 \mathrm{~mm}$ in Q1, 6.81$7.35 \mathrm{~mm}$ in Q2, 7.36-8.10 $\mathrm{mm}$ in Q3, and $>8.10 \mathrm{~mm}$ in Q4.

The highest quartile (CCA diameter $>8.10 \mathrm{~mm}$ ) had a 3.41 times (95\% CI: 1.96-5.92) greater risk than the lowest quartile (CCA diameter $\leq 6.80 \mathrm{~mm}$ ) in the crude model. The risk of stroke attenuated to 2.49 (95\% CI: 1.36-4.56) after adjusting for age, education level, household income, excess weight or obesity, current smoking habit, current drinking habit, lack of exercise, diabetes, and dyslipidemia ( $\mathrm{P}$ for trend $<0.05$ ). Among the men, the CCA diameters were $\leq 7.10 \mathrm{~mm}$ in Q1, 7.11-7.70 $\mathrm{mm}$ in Q2, 7.71-8.45 $\mathrm{mm}$ in $\mathrm{Q} 3$, and $>8.45 \mathrm{~mm}$ in Q4. However, we found no significant trends across the quartiles in men.

\section{$C-R$ relationship between $C C A$ diameter and the risk of stroke}

The C-R relationship was analyzed to assess the association between CCA diameter and the risk of stroke (Figure 2). In this plot, we found a positive nonlinear relationship between CCA diameter and the risk of stroke in women. This result further confirmed the logistic regression analysis.

\section{Incremental ability of CCA diameter to optimize the risk stratification of stroke}

Reclassification analysis was conducted to exam the incremental value of the CCA diameter in optimizing stroke risk stratification in men and women (Table 3). In women, we found a significant category-free NRI $(0.325$; 95\% CI: $0.173-0.476 ; \mathrm{P}<0.001)$ and IDI $(0.008 ; 95 \% \mathrm{CI}$ : $0.004-0.012 ; \mathrm{P}<0.001)$ when introducing $\mathrm{CCA}$ diameter as a clinical risk factor for the reclassification analysis. However, no significant difference was found in men when we introduced the CCA diameter as a clinical risk factor.

\section{Discussion}

In the present study, we observed a sex-specific relationship 
Table 1 Baseline characteristics of the study participants $(\mathrm{N}=3,245)$

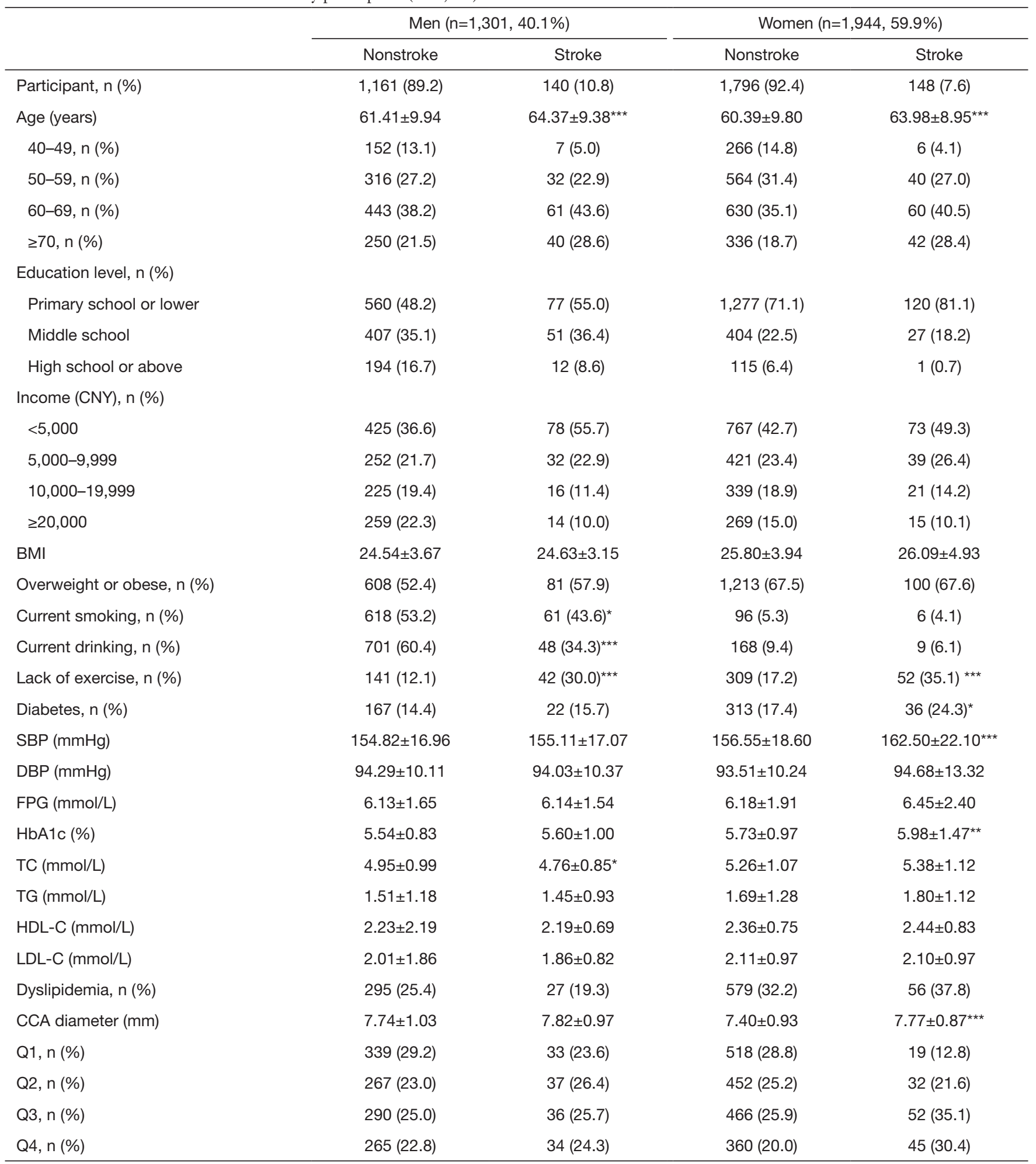

${ }^{*} \mathrm{P}<0.05$, ${ }^{*} \mathrm{P}<0.01,{ }^{* *} \mathrm{P}<0.001$ versus nonstroke group in both men and women. Data are expressed as mean \pm standard deviation (SD) or median (interquartile range) and numbers (percentage) as appropriate. CNY: Chinese yuan (1CNY=0.15 USD); BMI, body mass index; SBP, systolic blood pressure; DBP, diastolic blood pressure; FPG, fasting plasma glucose; TC, total cholesterol; TG, triglyceride; HDL-C, high-density lipoprotein cholesterol; LDL-C, low-density lipoprotein cholesterol. 
Table 2 Associations between CCA diameter and stroke in multiple logistic models after adjustment for cerebral vascular risk factors

\begin{tabular}{|c|c|c|c|c|}
\hline Model & \multicolumn{4}{|c|}{ OR $(95 \% \mathrm{Cl})$} \\
\hline \multicolumn{5}{|c|}{ Quartile of CCA diameter in men } \\
\hline Model 1 & $1.42(0.87-2.34)$ & $1.28(0.78-2.10)$ & $1.32(0.80-2.18)$ & $1.08(0.92-1.26)$ \\
\hline Model 2 & $1.26(0.76-2.08)$ & $1.03(0.62-1.72)$ & $1.00(0.59-1.69)$ & $0.98(0.83-1.15)$ \\
\hline \multicolumn{5}{|c|}{ Quartile of CCA diameter in women } \\
\hline Model 1 & $1.93(1.08-3.45)^{\star}$ & $3.04(1.77-5.22)^{\star \star \star}$ & $3.41(1.96-5.92)^{\star \star \star}$ & $1.47(1.26-1.73)^{\star \star \star}$ \\
\hline Model 2 & $1.64(0.91-2.97)$ & $2.35(1.33-4.16)^{\star \star}$ & $2.52(1.38-4.60)^{\star *}$ & $1.34(1.12-1.60)^{\star \star \star}$ \\
\hline Model 3 & $1.64(0.90-2.98)$ & $2.41(1.36-4.26)^{\star \star}$ & $2.49(1.36-4.56)^{\star \star}$ & $1.34(1.12-1.60)^{\star \star *}$ \\
\hline
\end{tabular}

${ }^{*} \mathrm{P}<0.05,{ }^{*} \mathrm{P}<0.01,{ }^{* * *} \mathrm{P}<0.001$. Model 1: unadjusted; Model 2: adjusted for age; Model 3: adjusted for age, education level, income, excess weight or obesity, current smoking habit, current drinking habit, lack of exercise, diabetes, and dyslipidemia. Q: quartile; OR, odds ratio; CCA, common carotid artery.

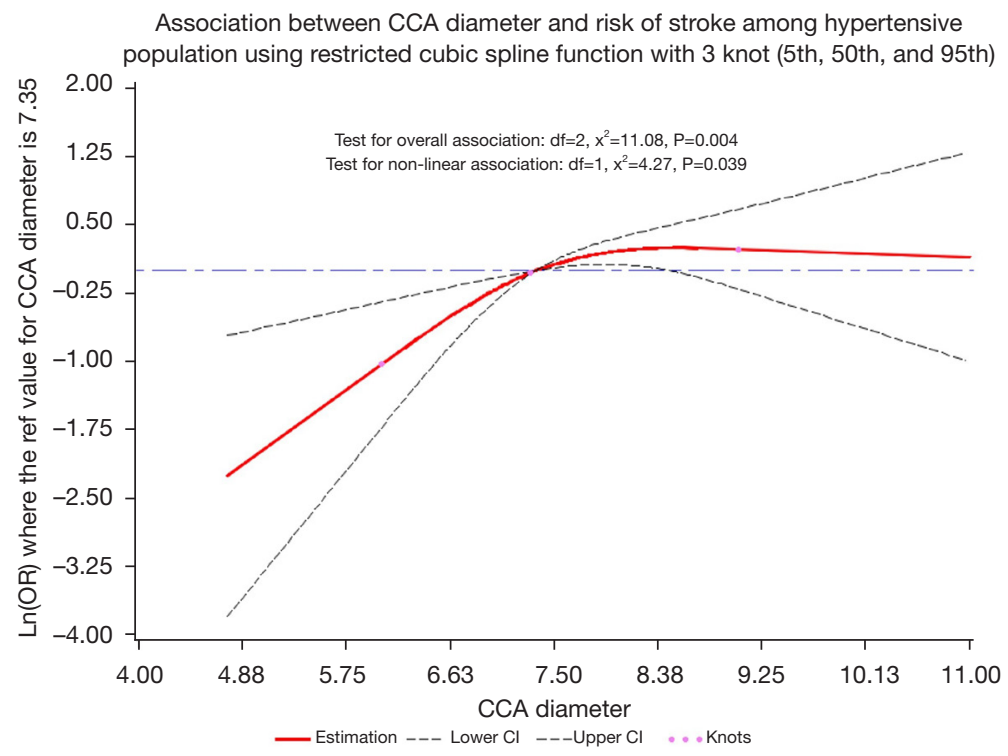

Figure 2 Concentration-response association between common carotid artery (CCA) diameter (mm) and stroke in hypertensive women after adjustments for age, education level, income, excess weight/obesity, current smoking habits, current drinking habits, lack of exercise, diabetes, and dyslipidemia. CCA diameter values were encoded using a linear model of a restrictive cubic spline (RCS) function with 3 knots located at the 5th, 50th, and 95th percentiles of CCA diameter distribution. Y-axis values show the changes of stroke prevalence between the indicated CCA diameter values and the reference value. The dashed lines are the $95 \%$ CIs.

between CCA diameter and the risk of stroke among individuals with hypertension. In the hypertensive study population, we found significant improvement in predictive ability when including CCA diameter as one of several major clinical risk factors in women, suggesting it has potential incremental value for optimizing the risk stratification of stroke in women.

Stroke is reported to be the most devastating manifestation of atherosclerosis and hypertension. Hypertension accounts for between $59.8 \%$ and $65.1 \%$ of the disability-adjusted life years lost due to stroke (20). Evidence consistently demonstrates a strong relationship 
Table 3 Incremental value of CCA diameter in predicting the probability of stroke

\begin{tabular}{lcccc}
\hline Variables & NRI $(95 \% \mathrm{Cl})$ & P value & IDI $(95 \% \mathrm{Cl})$ & $\mathrm{P}$ value \\
\hline CCA diameter & & & & \\
Men & $0.077(-0.091$ to 0.245$)$ & 0.37 & $0.001(-0.001$ to 0.004$)$ & 0.33 \\
Women & $0.325(0.173-0.476)$ & $<0.001$ & $0.008(0.004-0.012)$ & $<0.001$ \\
\hline
\end{tabular}

Reclassification indices were calculated for the addition of CCA diameter in the model adjusted for age, education level, income, excess weight or obesity, current smoking habit, current drinking habit, lack of exercise, diabetes, and dyslipidemia.

between high blood pressure, atherosclerosis, and the risk of stroke $(21,22)$. Management of these risk factors would significantly reduce the incidence of stroke and attenuate stroke-related cognitive impairment (23). There is thus an urgent need to deploy all possible measures to prevent atherosclerosis and stroke in the hypertensive population.

Unlike previous studies conducted in different countries $(7,24,25)$, the present study found CCA diameter to be independently associated with stroke prevalence in hypertensive Chinese women in particular, even after adjustments for traditional clinical risk factors were made. These findings suggest that arterial remodeling is an important factor in the risk of stroke in individuals with hypertension. Hypertension can independently trigger the process of atherogenesis at different stages (26). Large- and medium-sized artery dilatation is also an atherosclerotic process in response to chronically elevated blood pressures and is not only associated with aging $(10,27)$. Rupture of the load-bearing elastin fibers resulting from elevated tensile wall stress potentially contributes to arterial enlargement in a hypertensive population $(10,28)$. Moreover, large and elastic artery dilation is considered an adaptive process in response to the elevated afterload in individuals with hypertension (28). In addition, a previous study demonstrated that increased pulse pressure was associated with cyclic stretch, which might contribute to large, elastic artery dilation in hypertensive individuals (29). Therefore, compensatory carotid artery enlargement has been considered to be a valid predictor of adverse cardiovascular events (24), especially in women.

A pooled analysis that examined 4,887 participants from 4 cohort studies found that individuals in the top quartile of CCA diameter $(>8.10 \mathrm{~mm})$ had a higher incidence of stroke [odds ratio (OR) $1.5 ; 95 \%$ CI: 1.1-2.0] when compared to the bottom quartile (diameter $\leq 6.80 \mathrm{~mm}$ ) after adjusting for cardiovascular risk factors. However, the study population included participants of different ethnicities (7), and ethnic differences in CCA diameter and carotid intima-media thickness (CIMT) have been confirmed (11). In our study of a Chinese hypertensive population, we found the highest quartile of CCA diameter $(>8.10 \mathrm{~mm})$ had a 2.49 times $(95 \%$ CI: 1.36-4.56) greater risk of stroke than did the lowest quartile (diameter $\leq 6.80 \mathrm{~mm}$ ) in a fully adjusted model for women, indicating the potential incremental value of CCA diameter in improving risk stratification in hypertensive women.

The sex-specific association between CCA diameter and stroke risk in the present study could be partially explained by different risk profiles for atherosclerosis and stroke in men and women. According to our previous studies, men in northeast China were more likely to have multiple cardiovascular risk factors, such as a current smoking habit, excess weight, or obesity $(12,15,30)$. The interaction between risk factors might have a complicated effect on the atherosclerotic process. Although the diameters in male nonstroke populations were higher than those in female nonstroke populations in our study, the ages in these 2 groups also presented significant differences. Therefore, a larger number of participants might be needed to further identify differences. In addition, a previous study demonstrated greater stiffening and enlargement of the carotid arteries in older women, possibly due to higher afterload (31). The differences arising from the age-sex interaction in men and women may also explain the sexrelated disparities in the present study.

Sex-related disparities in stroke and risk factors have been reported previously (8). Women tend to have a higher lifetime risk and are more likely to be adversely affected by stroke than men, especially in high-income countries (32). Although the strength of the associations between stroke and risk factors are similar in men and women, the frequencies of risk factors differ between the sexes (33). The heavy burden of stroke in women calls for greater consideration of stroke in women's health (33). Therefore, identifying women at higher risk of stroke and formulating appropriate prevention strategies are critically 
important. Our study suggested the value of CCA diameter in optimizing stroke risk stratification in a hypertensive population, providing a valid monitoring index to help reduce the incidence of stroke in women.

The strengths of the study include its large populationbased design in a Chinese hypertensive population and a comprehensive estimation of the relationship between CCA diameter and stroke. However, the study had several limitations. First, we could only clarify association between CCA diameter and stroke prevalence, but the causality of this relationship still needs to be determined by further longitudinal studies. Second, our population originated from the rural areas of northeast China, and whether our results are transferable to patients of different ethnicity and economic status also needs to be confirmed in further studies.

\section{Conclusions}

The present study demonstrated the potential relationship between CCA diameter and stroke prevalence in a Chinese hypertensive population. We demonstrated the positive associations in the full range of CCA diameter in women, indicating that CCA diameter might be a possible predictor of stroke in women with hypertension. Furthermore, our results suggested the potential incremental value of CCA diameter in optimizing the risk stratification and prevention of stroke, which might provide a cost-effective and simple solution to alleviate the stroke burden in women.

\section{Acknowledgments}

Funding: This study was supported by Liaoning Revitalization Talents Program (XLYC2007058), the National Natural Science Foundation of China (62171472), the Natural Science Foundation of Liaoning (2021-MS171), and the Department of Science and Technology of Liaoning Province (2019JH2/10300001, 2018225065).

\section{Footnote}

Reporting Checklist: The authors have completed the STROBE reporting checklist. Available at https://dx.doi. org/10.21037/qims-21-598

Conflicts of Interest: All authors have completed the ICMJE uniform disclosure form (available at https://dx.doi. org/10.21037/qims-21-598). The authors report that they performed the study by the support of the fundings (Liaoning Revitalization Talents Program (XLYC2007058), the National Natural Science Foundation of China (62171472), Natural Science Foundation of Liaoning (2021-MS-171), Department of Science and Technology of Liaoning Province (2019JH2/10300001, 2018225065)).

Etbical Statement: The authors are accountable for all aspects of the work in ensuring that questions related to the accuracy or integrity of any part of the work are appropriately investigated and resolved. The study was conducted in accordance with the Declaration of Helsinki (as revised in 2013). The study protocol was approved by the central ethics committee of China National Center for Cardiovascular Disease. Written consents were obtained from all participants.

Open Access Statement: This is an Open Access article distributed in accordance with the Creative Commons Attribution-NonCommercial-NoDerivs 4.0 International License (CC BY-NC-ND 4.0), which permits the noncommercial replication and distribution of the article with the strict proviso that no changes or edits are made and the original work is properly cited (including links to both the formal publication through the relevant DOI and the license). See: https://creativecommons.org/licenses/by-nc-nd/4.0/.

\section{References}

1. GBD 2016 Mortality Collaborators. Global, regional, and national under-5 mortality, adult mortality, age-specific mortality, and life expectancy, 1970-2016: a systematic analysis for the Global Burden of Disease Study 2016. Lancet 2017;390:1084-150.

2. Feigin VL, Roth GA, Naghavi M, Parmar P, Krishnamurthi R, Chugh S, Mensah GA, Norrving B, Shiue I, Ng M, Estep K, Cercy K, Murray CJL, Forouzanfar MH; Global Burden of Diseases, Injuries and Risk Factors Study 2013 and Stroke Experts Writing Group. Global burden of stroke and risk factors in 188 countries, during 1990-2013: a systematic analysis for the Global Burden of Disease Study 2013. Lancet Neurol 2016;15:913-24.

3. Cipolla MJ, Liebeskind DS, Chan SL. The importance of comorbidities in ischemic stroke: Impact of hypertension on the cerebral circulation. J Cereb Blood Flow Metab 2018;38:2129-49.

4. Cuspidi C, Sala C, Tadic M, Rescaldani M, De Giorgi 
GA, Grassi G, Mancia G. Untreated masked hypertension and carotid atherosclerosis: a meta-analysis. Blood Press 2015;24:65-71.

5. Li F, Yang L, Yang R, Xu W, Chen FP, Li N, Zhang JB. Ischemic Stroke in Young Adults of Northern China: Characteristics and Risk Factors for Recurrence. Eur Neurol 2017;77:115-22.

6. Ward MR, Pasterkamp G, Yeung AC, Borst C. Arterial remodeling. Mechanisms and clinical implications. Circulation 2000;102:1186-91.

7. Sedaghat S, van Sloten TT, Laurent S, London GM, Pannier B, Kavousi M, Mattace-Raso F, Franco OH, Boutouyrie P, Ikram MA, Stehouwer CDA. Common Carotid Artery Diameter and Risk of Cardiovascular Events and Mortality: Pooled Analyses of Four Cohort Studies. Hypertension 2018;72:85-92.

8. Barker-Collo S, Bennett DA, Krishnamurthi RV, Parmar P, Feigin VL, Naghavi M, Forouzanfar MH, Johnson CO, Nguyen G, Mensah GA, Vos T, Murray CJ, Roth GA; GBD 2013 Stroke Panel Experts Group. Sex Differences in Stroke Incidence, Prevalence, Mortality and DisabilityAdjusted Life Years: Results from the Global Burden of Disease Study 2013. Neuroepidemiology 2015;45:203-14.

9. Seshadri S, Beiser A, Kelly-Hayes M, Kase CS, Au R, Kannel WB, Wolf PA. The lifetime risk of stroke: estimates from the Framingham Study. Stroke 2006;37:345-50.

10. Yang Y, Wang Y, Xu J, Gao P. Association between common carotid artery diameter and target organ damage in essential hypertension. J Hypertens 2018;36:537-43.

11. Markert MS, Della-Morte D, Cabral D, Roberts EL Jr, Gardener H, Dong C, Wright CB, Elkind MS, Sacco RL, Rundek T. Ethnic differences in carotid artery diameter and stiffness: the Northern Manhattan Study. Atherosclerosis 2011;219:827-32.

12. Xing L, Jing L, Tian Y, Liu S, Lin M, Du Z, Ren G, Sun Q, Shi L, Dai D, Liu S. High prevalence of stroke and uncontrolled associated risk factors are major public health challenges in rural northeast China: A population-based study. Int J Stroke 2020;15:399-411.

13. American Psychiatric Association. Diagnostic and statistical manual of mental disorder, 5th ed. Text Revision (DSM-5). Washington, DC: American Psychiatric Association; 2013.

14. Liu LS; Writing Group of 2010 Chinese Guidelines for the Management of Hypertension. 2010 Chinese guidelines for the management of hypertension. Zhonghua Xin Xue Guan Bing Za Zhi 2011;39:579-615.

15. Xing L, Jing L, Tian Y, Yan H, Zhang B, Sun Q, Dai D, Shi L, Liu D, Yang Z, Liu S. Epidemiology of dyslipidemia and associated cardiovascular risk factors in northeast China: A cross-sectional study. Nutr Metab Cardiovasc Dis 2020;30:2262-70.

16. Shi W, Xing L, Jing L, Tian Y, Yan H, Sun Q, Dai D, Shi L, Liu S. Value of triglyceride-glucose index for the estimation of ischemic stroke risk: Insights from a general population. Nutr Metab Cardiovasc Dis 2020;30:245-53.

17. Hatano S. Experience from a multicentre stroke register: a preliminary report. Bull World Health Organ 1976;54:541-53.

18. Wang Y, Li Z, Wang Y, Zhao X, Liu L, Yang X, Wang C, Gu H, Zhang F, Wang C, Xian Y, Wang DZ, Dong Q, Xu A, Zhao J. Chinese Stroke Center Alliance: a national effort to improve healthcare quality for acute stroke and transient ischaemic attack: rationale, design and preliminary findings. Stroke Vasc Neurol 2018;3:256-62 .

19. Xing L, Liu S, Tian Y, Yan H, Jing L, Chen K, Yan F, Li Y, Lv J, Sun Y. C-R Relationship between Fasting Plasma Glucose and Unfavorable Outcomes in Patients of Ischemic Stroke withoutDiabetes. J Stroke Cerebrovasc Dis 2019;28:1400-8.

20. Pandian JD, Gall SL, Kate MP, Silva GS, Akinyemi RO, Ovbiagele BI, Lavados PM, Gandhi DBC, Thrift AG. Prevention of stroke: a global perspective. Lancet 2018;392:1269-78.

21. Gentil A, Béjot Y, Lorgis L, Durier J, Zeller M, Osseby GV, Dentan G, Beer JC, Moreau T, Giroud M, Cottin Y. Comparative epidemiology of stroke and acute myocardial infarction: the Dijon Vascular project (Diva). J Neurol Neurosurg Psychiatry 2009;80:1006-11.

22. Lv P, Ji A, Zhang R, Guo D, Tang X, Lin J. Circumferential degree of carotid calcification is associated with new ischemic brain lesions after carotid artery stenting. Quant Imaging Med Surg 2021;11:2669-76.

23. Sharma M, Hakim AM. The management of hypertension for primary stroke prevention: a proposed approach. Int J Stroke 2011;6:144-9.

24. Polak JF, Sacco RL, Post WS, Vaidya D, Arnan MK, O'Leary DH. Incident stroke is associated with common carotid artery diameter and not common carotid artery intima-media thickness. Stroke 2014;45:1442-6.

25. Eigenbrodt ML, Evans GW, Rose KM, Bursac Z, Tracy RE, Mehta JL, Couper DJ. Bilateral common carotid artery ultrasound for prediction of incident strokes using intima-media thickness and external diameter: an observational study. Cardiovasc Ultrasound 2013;11:22.

26. Hurtubise J, McLellan K, Durr K, Onasanya O, Nwabuko D, Ndisang JF. The Different Facets of Dyslipidemia and 
Hypertension in Atherosclerosis. Curr Atheroscler Rep 2016;18:82.

27. Lam CS, Xanthakis V, Sullivan LM, Lieb W, Aragam J, Redfield MM, Mitchell GF, Benjamin EJ, Vasan RS. Aortic root remodeling over the adult life course: longitudinal data from the Framingham Heart Study. Circulation 2010;122:884-90.

28. Crouse JR, Goldbourt U, Evans G, Pinsky J, Sharrett AR, Sorlie P, Riley W, Heiss G. Risk factors and segment-specific carotid arterial enlargement in the Atherosclerosis Risk in Communities (ARIC) cohort. Stroke 1996;27:69-75.

29. Boutouyrie P, Bussy C, Lacolley P, Girerd X, Laloux B, Laurent S. Association between local pulse pressure, mean blood pressure, and large-artery remodeling. Circulation 1999;100:1387-93.

Cite this article as: Xue W, Tian Y, Jing L, Li G, Yan H, Zhang B, Xing L, Liu S. Sex-specific prediction value of common carotid artery diameter for stroke risk in a hypertensive population: a cross-sectional study. Quant Imaging Med Surg 2022;12(2):1428-1437. doi: 10.21037/qims-21-598
30. Xing L, Jing L, Tian Y, Wang W, Sun J, Jiang C, Shi L, Dai D, Liu S. Epidemiology of stroke in urban northeast China: A population-based study 2018-2019. Int J Stroke 2021;16:73-82.

31. Vaidya D, Heckbert SR, Wasserman BA, Ouyang P. Sexspecific association of age with carotid artery distensibility: multi-ethnic study of atherosclerosis. J Womens Health (Larchmt) 2012;21:516-20.

32. Bushnell C, McCullough LD, Awad IA, Chireau MV, Fedder WN, Furie KL, et al. Guidelines for the prevention of stroke in women: a statement for healthcare professionals from the American Heart Association/ American Stroke Association. Stroke 2014;45:1545-88.

33. Kaplovitch E, Anand SS. Stroke in Women: Recognizing Opportunities for Prevention and Treatment. Stroke 2018;49:515-7. 